\title{
Comparison of three methods for reconstructing 3D motion from 2D video recordings for low cost gait analysis systems
}

\author{
Szymon CYGANa, ${ }^{*}$, Adriana SPECYALSKA ${ }^{b}$ \\ ${ }^{a}$ Institute of Metrology and Biomedical Engineering, Warsaw University of Technology, Poland \\ ${ }^{b}$ Modulo Studio Treningu EMS, Poland \\ ${ }^{*}$ E-mail address: szymon.cygan@pw.edu.pl
}

\begin{abstract}
Purpose: The aim of this study was to quantify the accuracy of 3D trajectory reconstructions performed from two planar video recordings, using three different reconstruction methods. Additionally, the recordings were carried out using easily available equipment, like built-in cellphone cameras, making the methods suitable for low-cost applications.

Methods: A setup for 3D motion tracking was constructed and used to acquire 2D video recordings subsequently used to reconstruct the 3D trajectories by 1) merging appropriate coordinates, 2) merging coordinates with proportional scaling, and 3) calculating the 3D position based on markers' projections on the viewing plane. As experimental verification, two markers moving at a fixed distance of $98.9 \mathrm{~cm}$ were used to assess the consistency of results. Next, gait analysis in five volunteers was carried out to quantify the differences resulting from different reconstruction methods.

Results: Quantitative evaluation of the investigated 3D trajectories reconstruction methods showed significant differences between those methods, with the worst reconstruction approach resulting in a maximum error of $50 \%$ (standard deviation $13 \%$ ), while the best resulting in a maximum error of $1 \%$ (standard deviation $0.44 \%$ ). The gait analysis results showed differences in mean angles obtained with each reconstruction method reaching only $2^{\circ}$, which can be attributed to the limited measurement volume.

Conclusions: Reconstructing 3D trajectory from 2D views without accounting for the "perspective error" results in significant reconstruction errors. The third method described in this study enables a significant reduction of this issue. Combined with the proposed setup, it provides a functional, low-cost gait analysis system.
\end{abstract}

Key words: gait analysis; 3D motion tracking; template matching.

\section{Introduction}

Human motion tracking and analysis are performed for various purposes including medicine, sports and entertainment. ${ }^{1}$ In many cases it is carried out using advanced equipment in a dedicated and purposely prepared environment - laboratories or studios. Gait analysis for clinical applications is performed in such settings with motion tracking supported by ground reaction force measurements as well as with dynamic electromyography. ${ }^{2,3}$ Similar solutions are applied in sports biomechanics. ${ }^{4}$ In both fields, obtained kinetic and kinematic data is frequently used as input data for musculoskeletal simulations of the studied movements and events. ${ }^{4-6}$

There are various approaches and methods to motion tracking depending on applications. A systematic review of most of the systems was presented by van der Kruk and Reijne. ${ }^{1}$ Optoelectronic systems with passive markers are considered a gold standard in most cases of motion capture applications. They enable the registration of 3D motion of markers placed in anatomically characteristic points of the body. Recently, marker-less systems are also being developed, enabling the acquisition of the whole body surface, but the large amount of data acquired and the need to identify characteristic landmarks have been limiting their capabilities. ${ }^{\mathbf{1} 7-9}$ A promising tool for overcoming this problem is the application of artificial intelligence (i.e. deep learning) methods for identification or rather recognition of objects and/or landmarks (i.e. body parts) visible in the frame and thus their tracking in the video sequence. A recent and extensive review on the subject was published in reference ${ }^{\mathbf{1 0}}$. Machine learning has been also successfully applied to the interpretation of gait analysis data for diagnostic purposes. ${ }^{11}$

Beside the advanced, 'high-end' solutions, simple methods have been proposed, enabling various motion tracking applications without expensive equipment. ${ }^{12-14}$ In this work we investigate the accuracy of three different approaches to 3D trajectory reconstruction from two video recordings. The presented approaches can be easily implemented in various environments and are suitable for low-cost systems. We expect that reconstruction of 3D coordinates by the simple merging of the $2 \mathrm{D}$ coordinates should introduce estimation errors, which can be significantly reduced by combining the information from both views. 


\section{Materials and Methods}

\section{Motion tracking and analysis method}

The proposed 3D motion tracking and analysis method, implemented in Matlab, uses two 2D video recordings registered synchronically with two cameras, placed perpendicularly to each other (Figure 1 and Figure 2). In order to analyse the activity, the volunteer has to be equipped with markers (marked as $P_{m}$ in Figure 1 and Figure 2) attached to the selected landmarks on the body. Furthermore, the markers have to be distinctively visible in both views during the whole recording.

The general flow of the proposed motion tracking and analysis method is shown in Figure 3. In the first step, the two recordings must be synchronised. In this study, it was done semiautomatically based on a single flash of light registered in the beginning of the recordings allowing for temporal alignment of both videos by matching frames with maximum intensities.

In the second step, the user identifies each marker in the first frame of both videos by pointing them with a cursor. This requires, that for every application an appropriate set of markers is predefined. For this study, two sets were used - for the quantitative assessment of tracking accuracy, the user was prompted for pointing two markers - "upper" and "lower". In the case of gait analysis markers for landmarks had to be indicated on the images - toe, ankle, knee, hip. Based on this action a template for each marker and each view is created covering the marker and its nearest surrounding (in this case template $25 \times 25$ pixels was used).

All the identified markers are tracked using a template matching method ${ }^{\mathbf{1 5}}$ through the whole video sequence. The template matching searches for a location on subsequent frames, where the template matches best using the sum of absolute differences as the similarity measure. ${ }^{16}$ The search is performed in a limited search region. The size of this region is calculated for each video using Equation 1, based on the maximum allowed/expected velocity $v_{\max }$, image resolution res and video frame rate fps.

$\operatorname{range}[p x l]=\frac{v_{\max }\left[\frac{m}{s}\right] * r e s\left[\frac{p x l}{m}\right]}{f p s\left[\frac{1}{s}\right]}$

Eq. 1

The resolution res in pixels per meter is valid only for the $y z$ and $x z$ planes and is calculated for each recording based on the distance between two selected markers measured at the preparation stage.
As a result of this tracking 2D trajectories of the projections of all the markers onto the $x z$ and $y z$ planes are obtained. Based on a measurement of the actual distance between selected markers, carried out at the subject preparation stage, both views can be scaled to meters. This scaling is assumed to be valid only for the nominal imaging plane e.g. in the $x z$ and $y z$ planes as shown in Figure 2 and is done based on the distance between two selected markers as mentioned above.
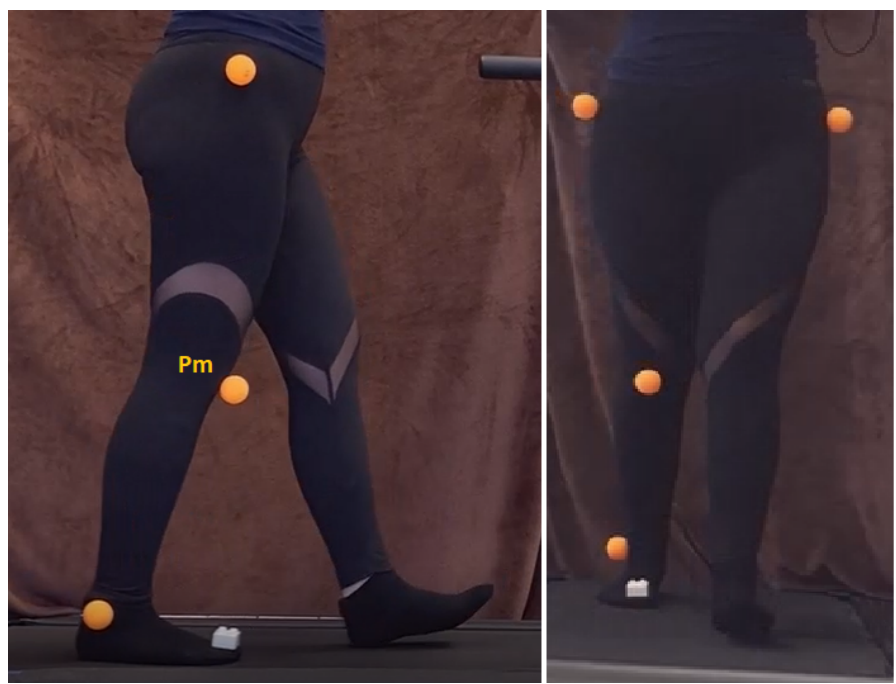

Figure 1. Sample frames from simultaneous recordings in sagittal (left) and coronal (right) planes.

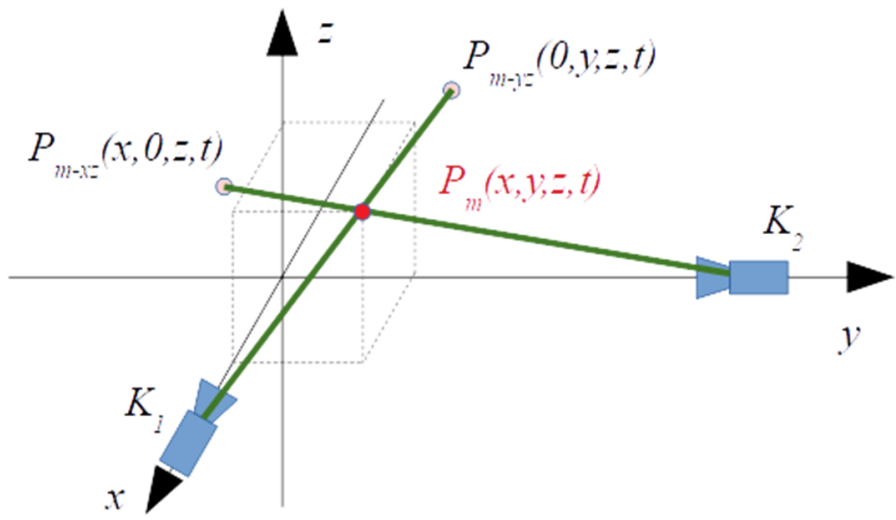

Figure 2. Schematic of the two camera $\left(K_{1}\right.$ and $\left.K_{2}\right)$ video registration setup with one marker $P_{m}$ visible.

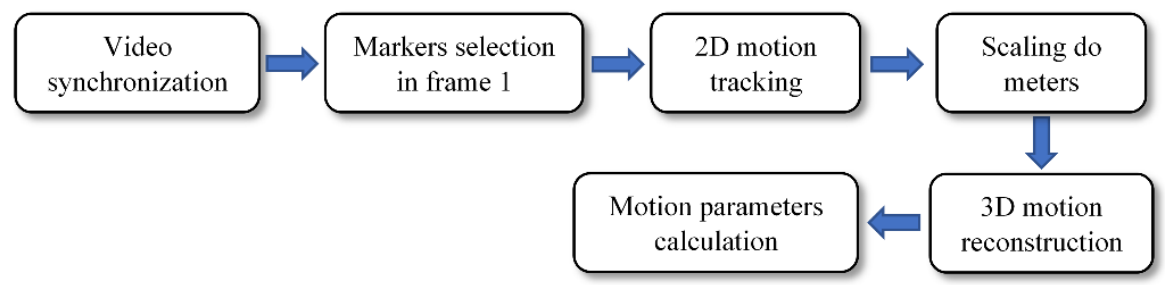

Figure 3. Algorithm of the motion analysis method. 
The reconstruction of $3 \mathrm{D}$ marker trajectory $P_{m}(x, y, z, t)$ was carried out in this study using three different approaches:

- By simple merging of $y$ and $z$ coordinates from the $K_{2}$ camera view and $x$ from the $K_{l}$ camera view, which will be referred as the merging method,

- By merging the abovementioned coordinates from separate views with proportional scaling of $y$ and $z$ based on the value of $x$ and vice versa, referred to as the proportional method. This method is based on the assumption that the scaling can be corrected for each view based on the information on the actual distance of the marker from the camera. This information is provided in the second view, so the corrected $x^{\prime}$ ( $t$ ) position of the marker is obtained by scaling the $x(t)$ based on the $y(t)$ coordinate and the position of the cameras $K_{l}$ and $K_{2}$ as given by the Equation 2. Other coordinates are corrected similarly.

$x^{\prime}(t)=x(t) \frac{\left(1-\frac{y(t)}{K_{2}}\right) * K_{1} * K_{2}}{K_{1} * K_{2}-x(t) * y(t)}$

Eq. 2

- By calculating the subsequent positions of the markers $P_{m}(x, y, z, t)$ as the locations of the intersections of the straight lines defined at each time step $t$ by respective cameras points $K_{I}\left(x_{K 1}, 0,0, t\right)$ and $K_{2}\left(0, y_{K 2}, 0, t\right)$ and the projection of the marker on the $x z$ or $y z$ plane: $P_{m-x z}(x, 0, z, t)$ and $P_{m-y z}(0, y, z, t)$ (Figure 2). This method is referred as the projections method. Since in the case of real data, the lines do not intersect ideally, instead of their intersection points, their nearest points $P_{m-s}$ and $P_{m-c}$ are identified (Figure 4) as well as the distance between them. The position of the marker $P_{m}(x, y, z, t)$ is the point lying halfway between the two points. This step is carried out using a Matlab function described in reference ${ }^{17}$.

Having obtained the 3D trajectories of the markers registered in the recordings, the necessary parameters of motion can be calculated - distances between points, amplitudes in each direction, angles between lines defined by two markers each etc. The calculation of angles between two lines at a given time instant or the change of slope of a single line is carried out using Equation 3, where the first line is defined by points $p 1$ and $p 2$ and the second by $r 1$ and $r 2$.

$\alpha=\frac{180^{\circ}}{\pi} \cdot\left(\operatorname{arctg} \frac{y_{r 1}-y_{r 2}}{x_{r 1}-x_{r 2}}-\operatorname{arctg} \frac{y_{p 1}-y_{p 2}}{x_{p 1}-x_{p 2}}\right)$

\section{Experimental data acquisitions}

In order to validate and test the proposed method and the three approaches to reconstructing the $3 \mathrm{D}$ trajectory from $2 \mathrm{D}$ recordings, two types of experimental data were registered. First the movement of two markers fixed at a constant distance of $99 \mathrm{~cm}$. Two orange spheres (diameter $40 \mathrm{~mm}$, weight $2.7 \mathrm{~g}$ ) were used as markers, covered with black rubber to be better distinguishable against a bright background (Figure 5). The movement was carried out in a wide measurement volume of approx. $150 \mathrm{~cm} \times 150 \mathrm{~cm} \times 120 \mathrm{~cm}$. The purpose of this experiment was to test the accuracy of the proposed method. The video data was registered using two built-in smartphone cameras (Samsung Galaxy S7 and Samsung Galaxy S5), each placed at a distance of $300 \mathrm{~cm}$ from the centre of the measurement volume, $83 \mathrm{~cm}$ above the floor. Perpendicular placement of the cameras was verified by measurement of the distance between cameras themselves which was $300 \mathrm{~cm} \times \sqrt{2}=424 \mathrm{~cm}$. Videos, $10 \mathrm{~s}$ long, were registered at resolution of 1080 x 1920 pixels with framerates of $30 \mathrm{fps}$.

The second set of data was recorded for 5 volunteers (4 female, 1 male) with mean age 26.2 y.o. (ranging 22 to 41 ), mean body height $174.2 \mathrm{~cm}$ (165 to 188), mean body weight $64 \mathrm{~kg}$ (53 to 77) walking on a treadmill (Kettler Track 3 ) with velocities of $2 \mathrm{~km} / \mathrm{h}, 3 \mathrm{~km} / \mathrm{h}$ and $4 \mathrm{~km} / \mathrm{h}$. The volunteers were asked to dress in tightly fitting, black clothing (Figure 1) and a dark background was provided. Five markers were placed on the body - four visible in both sagittal and frontal plane views, attached to second toe, ankle (lateral malleolus), knee (patella) and hip (iliac crest) and one visible only in the frontal plane view - on the opposite hip (Figure 1).

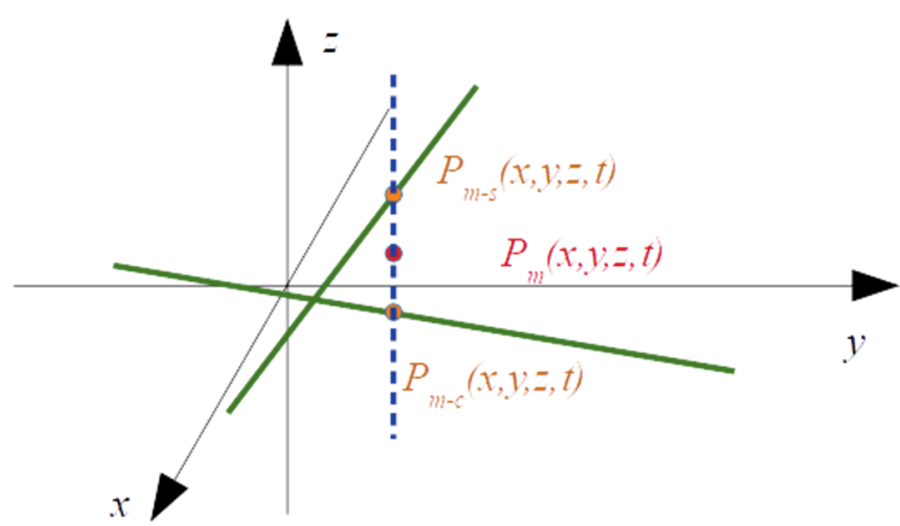

Figure 4. The estimated position of the marker as a point $\boldsymbol{P}_{m}$ halfway between both camera-marker projection lines (green).

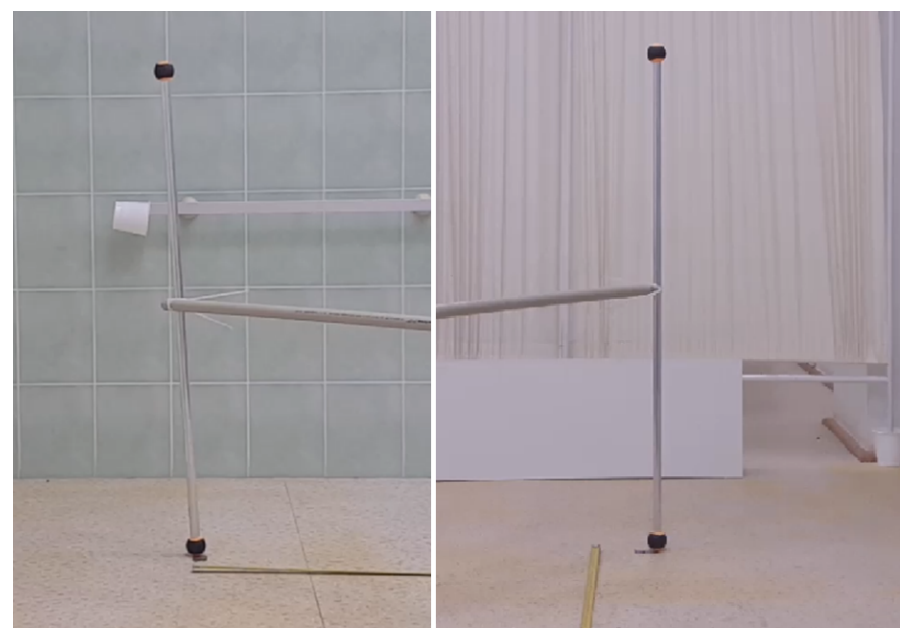

Figure 5. Frames from two simultaneous recordings of two markers at a fixed distance used for evaluation of the proposed methods. 
Again orange spheres of $40 \mathrm{~mm}$ in diameter along with one white cuboid block $(37.5 \mathrm{~mm} \times 37.5 \mathrm{~mm} \times 19 \mathrm{~mm})$ were used as markers. The following parameters were calculated from the recordings: angular range of motion in the ankle joint (ankle angle), angular range of motion in the knee joint (knee angle), the range of angular pelvic motion in the frontal plane (pelvis rocking) and the horizontal displacement of the pelvis in the frontal plane (pelvis displacement). Note that the marker placement was not done precisely according to the known and recommended standards. ${ }^{7,18}$ Measurements were carried out only in order to compare values between the three proposed 3D trajectory reconstruction methods and based on this assumption they were averaged for all participants. Each method was tested on the same set of all the data collected.

\section{Results}

\section{Quantitative accuracy assessment}

The nominal distance between markers, spaced vertically, was $98.9 \mathrm{~cm}$ in the video data used for quantitative accuracy assessment. The markers' movement range was $148.1 \mathrm{~cm} \times$ $147.7 \mathrm{~cm} \times 20.7 \mathrm{~cm}$, and the movement was irregular, as shown in Figure 6. The distance between the markers tracked using the proposed projections method throughout the whole data sequence was $99.1 \pm 0.44 \mathrm{~cm}(0.44 \%)$ with an error spread of $1.08 \mathrm{~cm}(1.09 \%)$, while for the merging method, it was $103 \pm 13$ $\mathrm{cm}(13.1 \%)$ with error spread of $55 \mathrm{~cm}(55 \%)$ as presented in Table 1.

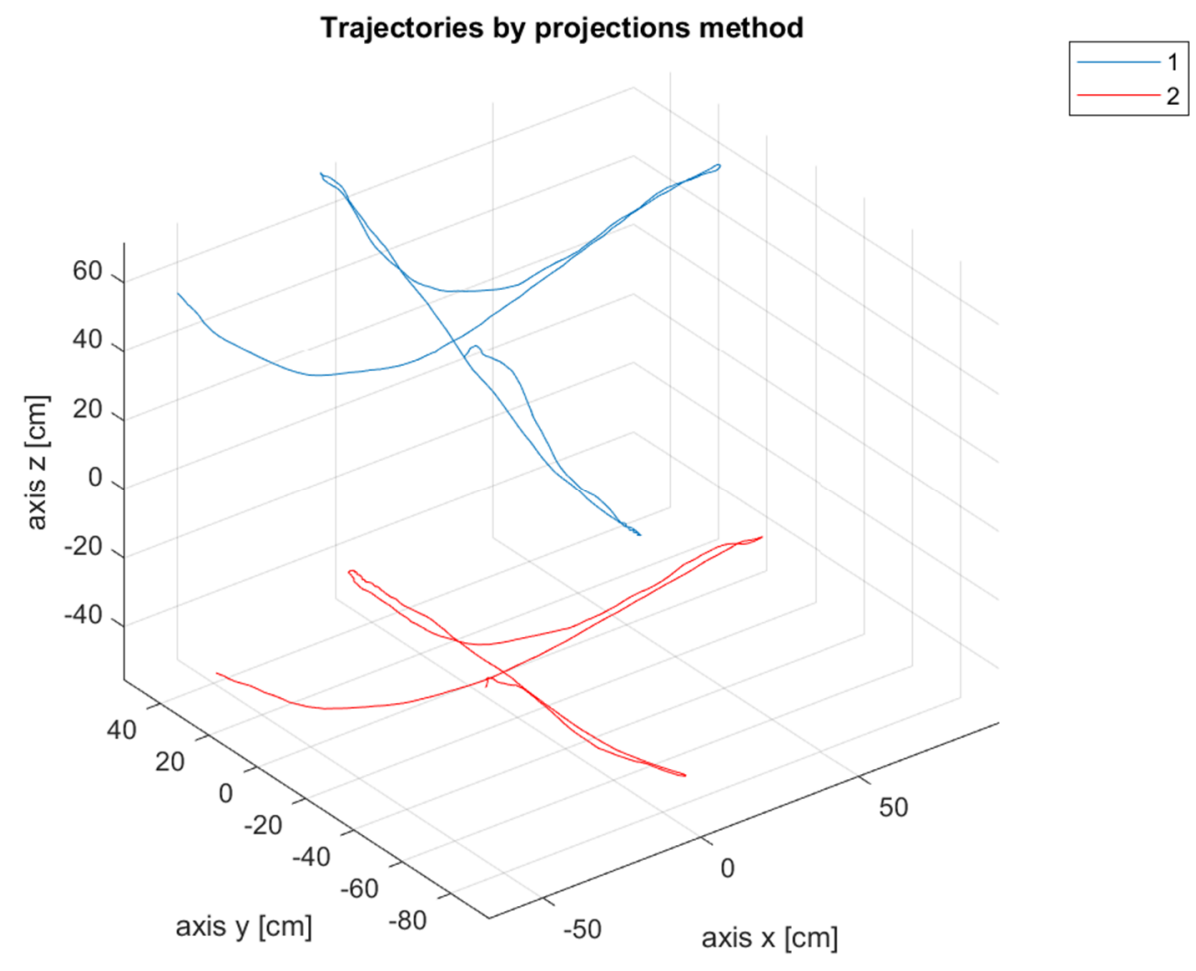

Figure 6. Trajectories of the two markers used for quantitative assessment of the proposed method obtained by the projections method.

Table 1. Quantitative assessment results for each 3D motion reconstruction method.

\begin{tabular}{lccc}
\hline \hline Method & Mean distance & Standard deviation & Maximum spread \\
\hline Merging & $103 \mathrm{~cm}$ & $13 \mathrm{~cm}$ & $55 \mathrm{~cm}$ \\
Proportional & $99.7 \mathrm{~cm}$ & $1.9 \mathrm{~cm}$ & $8.4 \mathrm{~cm}$ \\
Projections & $99.1 \mathrm{~cm}$ & $0.44 \mathrm{~cm}$ & $1.08 \mathrm{~cm}$ \\
\hline \hline
\end{tabular}




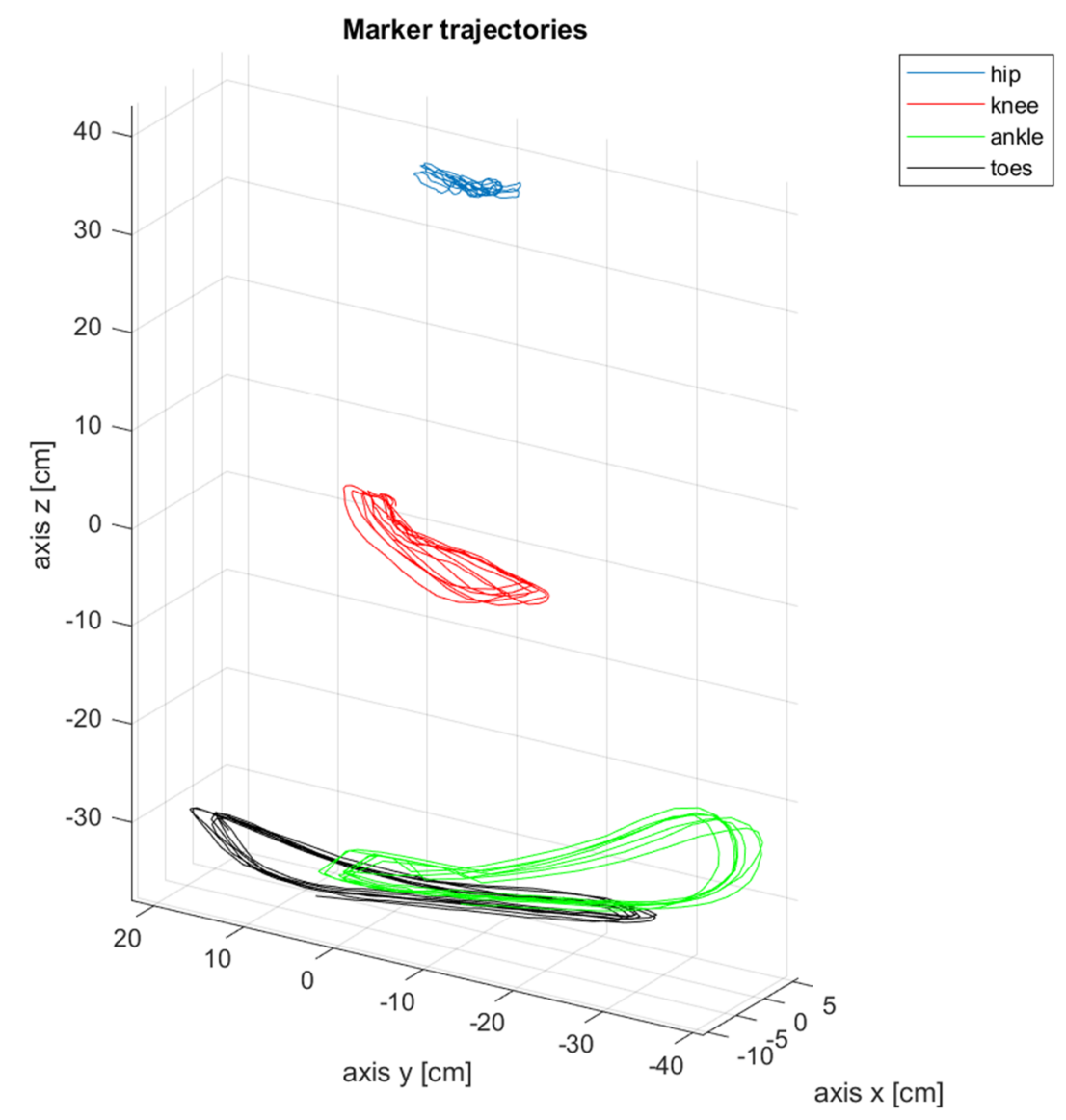

Figure 7. 3D plot of the trajectories obtained from one of the datasets registered with the volunteers.

Table 2. Gait analysis results for each 3D motion reconstruction method.

\begin{tabular}{|c|c|c|c|c|}
\hline Method & Ankle angle & Knee angle & Pelvis rocking & Pelvis displacement \\
\hline Merging & $27.2^{\circ} \pm 4^{\circ}$ & $49.1^{\circ} \pm 3.8^{\circ}$ & $9^{\circ} \pm 3.6^{\circ}$ & $5 \pm 1.4 \mathrm{~cm}$ \\
\hline Proportional & $29.2^{\circ} \pm 3.3^{\circ}$ & $50.7^{\circ} \pm 6^{\circ}$ & $7.3^{\circ} \pm 3.3^{\circ}$ & $4.6 \pm 1.3 \mathrm{~cm}$ \\
\hline Projections & $28.7^{\circ} \pm 3.5^{\circ}$ & $51.8^{\circ} \pm 4.9^{\circ}$ & $7.2^{\circ} \pm 3.7^{\circ}$ & $4.5 \pm 1.3 \mathrm{~cm}$ \\
\hline
\end{tabular}

\section{Gait analysis results}

In order to assess the influence of each 3D reconstruction method on the results of actual gait analysis, all volunteer-based data was used with the three methods. Three-dimensional trajectories of the movement of the markers were obtained (Figure 7). Ranges of motions averaged over all the cases obtained from the gait analysis using all three 3D trajectory reconstruction methods are presented in Table 2.

\section{Discussion}

\section{Accuracy assessment}

The experiment with two markers carried out for the purpose of quantitative accuracy assessment covered a relatively wide measurement area of approx. $150 \mathrm{~cm} \times 150 \mathrm{~cm} \times 120 \mathrm{~cm}$ and thus has shown the accuracy of the three methods for the reconstruction of 3D trajectories. Obtained results show large errors (low accuracy) of the simple method of merging the coordinates from the two views (Table 1). This method can provide accurate marker position only when the distance from the cameras remains unchanged - the marker stays in the centre of the coordinate system. When the distance from the camera changes, the scaling coefficient, i.e. resolution (res $[\mathrm{pxl} / \mathrm{m}]$ ) becomes invalid.

Results for the second approach - the proportional method (Table 1), where the coordinates are corrected based on the view from the other camera, show that it provided a significant improvement of the accuracy of tracking compared to the simple merging of coordinates method. This approach also merges coordinates from both views, but with additional correction of the perspective effect.

The last method, the projections-based reconstruction (projections method), showed the highest accuracy (Table 1). This was achieved by using the coordinates from each view as directional information rather than actual positions. A projection of the marker on the $x z$ or yz plane was tracked, and then it was assumed that the actual position of the marker was where the two viewing lines intersected. The improvement as compared to the second method - merging with proportional correction results from the use of all available information and its "averaging" by 
assuming that the actual marker lies halfway between the directional lines. This is also the standard photogrammetry triangulation method used for processing $2 \mathrm{D}$ data streams in contact-less passive marker-based motion capture systems. ${ }^{14}$

\section{Gait analysis}

The volunteer-based experiments showed that in the case of relatively small movement amplitudes that occur during gait analysis on a stationary treadmill, the errors introduced by insufficient compensation for the perspective are not as large as in the former experiment, but can still influence obtained results.

\section{Motion tracking method evaluation}

The proposed 3D, marker-based motion capture method in this simple form is susceptible to various sub-optimal data acquisition conditions. The most common problems are marker occlusion and marker swapping. The former is the situation when one of the markers is becoming not visible in one of the views, the latter occurs when two markers come close to one another in the image, and the algorithm wrongly identifies them. Handling of those problems has not been implemented in the proposed computational method, but has been taken into account to some extent. Each of those problems can be approached in different ways as described in reference ${ }^{19}$. The problem of occlusion has been addressed by enforcing an assumption on the data acquisition conditions requiring all the markers to be visible at all times (Table 1 in reference ${ }^{19}$ ), similarly as in the gold standard systems (i.e. Vicon), where each marker has to be visible to at least two cameras at all times. The problem of swapping can be addressed in various ways - at the stage of processing, by assuming rigidity of the body segments and their limited velocities. ${ }^{19}$ Here, during the gait analysis experiment a different solution at the stage of data acquisition was used to solve this problem. The two markers placed on the subject's foot were susceptible to swapping, and for this reason, different shapes and colours were used. Their different appearance allowed the template matching algorithm to identify the marker correctly.

\section{Conclusions}

This work shows that the proposed method enables 3D motion analysis to be carried out without specialised equipment and that it can be performed not only in the laboratory environment. When the projections method for 3D trajectories reconstruction is used, its accuracy fits in the wide range of accuracies reported for other available motion capture methods. ${ }^{1,20}$ In the presented implementation, the Matlab software used for data processing is the most expensive and specialised resource, but the same methods can be implemented for example in Python environment.

Accuracy of this method can be further improved without increasing its costs by increasing precision of markers selection, either by more careful manual selection in zoomed-in view or, by their segmentation in the data (region growing method is suitable for such case) and localising their centres (centroid localisation).

Another possible improvement method is the more precise setting of the measurement setup. Here the placement of cameras was done by hand with positions measured with a precision of close to $1 \mathrm{~cm}$.

\section{References}

1. van der Kruk E, Reijne MM. Accuracy of human motion capture systems for sport applications; state-of-the-art review. Eur J Sport Sci. 2018;18(6):806-819. https://doi.org/10.1080/17461391.2018.1463397

2. Syczewska M, Kocel K, Swiecicka A, et al. Selection of gait parameters for modified Gillette Gait Index using Hellwig Correlation Based Filter method, random forest method, and correlation methods. Biocybern Biomed Eng. 2020;40(3):1267-1276. https://doi.org/10.1016/J.BBE.2020.07.002

3. Łysoń-Uklańska B, Ścibek J, Bienias K, Wit A. Analysis of Ground Reaction Forces and Kinematic Response to Gait Perturbation During Mid- to Terminal Stance Phase of the Gait Cycle. Adv Intell Syst Comput. 2020;1223:165-173. https://doi.org/10.1007/978-3030-52180-6_19

4. Łysoń-Uklańska B, Błazkiewicz M, Kwacz M, Wit A. Muscle Force Patterns in Lower Extremity Muscles for Elite Discus Throwers, Javelin Throwers and Shot-Putters - A Case Study. J Hum Kinet. 2021;78(1):5-14. https://doi.org/10.2478/hukin-2021-0026

5. Groote F De, Falisse A. Perspective on musculoskeletal modelling and predictive simulations of human movement to assess the neuromechanics of gait. Proc R Soc B. 2021;288(1946). https://doi.org/10.1098/RSPB.2020.2432

6. Tecante K, Seehaus F, Welke B, et al. Clinical gait analysis and musculoskeletal modeling. In: Magnenat-Thalmann N, Ratib O, Choi H. (eds) 3D Multiscale Physiological Human. Springer, London. 2014. https://doi.org/10.1007/978-1-4471-6275-9_7

7. Colyer SL, Evans M, Cosker DP, Salo AIT. A Review of the Evolution of Vision-Based Motion Analysis and the Integration of Advanced Computer Vision Methods Towards Developing a Markerless System. Sport Med - Open. 2018;4(1):1-15. https://doi.org/10.1186/s40798-018-0139-y

8. Liberadzki P, Adamczyk M, Witkowski M, Sitnik R. Structured-light-based system for shape measurement of the human body in motion. Sensors (Switzerland). 2018;18(9). https://doi.org/10.3390/s18092827 
9. Desmarais Y, Mottet D, Slangen P, Montesinos P. A review of 3D human pose estimation algorithms for markerless motion capture. Comput Vis Image Underst. 2021;212:103275. https://doi.org/10.1016/J.CVIU.2021.103275

10. Mathis A, Schneider S, Lauer J, Mathis MW. A Primer on Motion Capture with Deep Learning: Principles, Pitfalls, and Perspectives. Neuron. 2020;108(1):44-65. https://doi.org/10.1016/J.NEURON.2020.09.017

11. Khera P, Kumar N. Role of machine learning in gait analysis: a review. Journal of Medical Engineering \& Technology. 2020;44(8):441467. https://doi.org/10.1080/03091902.2020.1822940

12. Magdin M. Simple MoCap System for Home Usage. Int J Interact Multimed Artif Intell. 2017;4(4):80. https://doi.org/10.9781/ijimai.2017.4410

13. Rajkiewicz P, Łepkowska K, Cygan S. Video markers tracking methods for bike fitting. In: Romaniuk RS, ed. Proceedings of SPIE The International Society for Optical Engineering. Vol 9662. International Society for Optics and Photonics; 2015:96621G. https://doi.org/10.1117/12.2199378

14. Kolahi A, Hoviattalab M, Rezaeian T, Alizadeh M, Bostan M, Mokhtarzadeh H. Design of a marker-based human motion tracking system. Biomed Signal Process Control. 2007;2(1):59-67. https://doi.org/10.1016/J.BSPC.2007.02.001

15. Chantara W, Mun J-H, Shin D-W, Ho Y-S. Object Tracking using Adaptive Template Matching. IEIE Trans Smart Process Comput. 2015;4(1):1-9. https://doi.org/10.5573/ieiespc.2015.4.1.001

16. Cygan S, Kaluzynski K, Lesniak B. Displacement estimation methods for elastography - a phantom study. Eng Mech. 2005;12(5):361368. http://www.engineeringmechanics.cz/pdf/12_5_361.a.pdf.

17. Shortest Distance Between Two Lines in $N$ dimensions - File Exchange - MATLAB Central. https://www.mathworks.com/matlabcentral/fileexchange/29130-shortest-distance-between-two-lines-in-n-dimensions. Accessed March 21, 2020.

18. Clark CCT, Barnes CM, Holton M, Summers HD, Stratton G. A Kinematic Analysis of Fundamental Movement Skills. Sport Sci Rev. 2016;25(3-4):261-275. https://doi.org/10.1515/ssr-2016-0014

19. Moeslund TB, Granum E. A Survey of Computer Vision-Based Human Motion Capture. Comput Vis Image Underst. 2001;81(3):231268. https://doi.org/10.1006/CVIU.2000.0897

20. Thewlis D, Bishop C, Daniell N, Paul G. Next-generation low-cost motion capture systems can provide comparable spatial accuracy to high-end systems. J Appl Biomech. 2013;29(1):112-117. https://doi.org/10.1123/jab.29.1.112 